Research Paper

\title{
Hepatitis B Virus X Protein Driven Alpha Fetoprotein Expression to Promote Malignant Behaviors of Normal Liver Cells and Hepatoma Cells
}

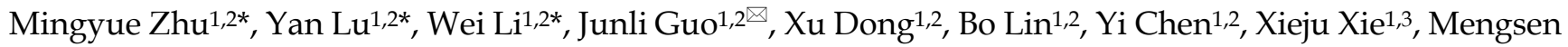
$\mathrm{Li}^{1,2,4}$

1. Hainan Provincial Key Laboratory of Carcinogenesis and Intervention, Hainan Medical College, Haikou 571199, Hainan Province, PR. China

2. Key Laboratory of Molecular Biology, Hainan Medical College, Haikou 571199, PR. China

3. Department of Pathophysiology, Hainan Medical College, Haikou 571199, Hainan Province, PR. China

4. Institution of Tumor, Hainan Medical College, Haikou 570102, Hainan Province, PR. China.

*These authors contribute equally to this work and are co-first authors.

$\triangle$ Corresponding authors: Mengsen Li and Junli Guo, Address: Hainan Provincial Key Laboratory of Carcinogenesis and Intervention, Hainan Medical College, 3 Xueyuan Road, Longhua District, Haikou 571199, Hainan Province, P.R.China. Tel: +86-898-66895322; Fax: +86-898-66895322; Email: mengsenli@163.com.

(c) Ivyspring International Publisher. Reproduction is permitted for personal, noncommercial use, provided that the article is in whole, unmodified, and properly cited. See http://ivyspring.com/terms for terms and conditions.

Received: 2015.08.21; Accepted: 2016.03.15; Published: 2016.05.12

\begin{abstract}
Background: The infection of Hepatitis B virus (HBV) is closely associated with the development of hepatocellular carcinoma $(\mathrm{HCC}), \mathrm{HBV}-\mathrm{X}$ protein $(\mathrm{HBx})$ is able to induce expression of alpha-fetoprotein(AFP) in normal liver cells, and AFP harbors a function to promote malignant transformation of normal liver cells, but the role AFP playing in malignant behaviors of HCC cells is still unclear.

Methods: Fifty-six liver tissue samples were collected from the clinical patients through hepatectomy(include normal liver tissues, HBV-related hepatitis liver tissues and HBV-related HCC tissues), and diagnosis of these tissues by pathology section, expression of AFP, Ras and CXCR4 were evidenced by immunohisochemical staining and Western blotting; The proliferation of human normal liver cells line L-02 cells and human hepatoma cells line, HLE cells(non AFP-producing) were performed by MTT method; Repaired capacity of L-02 and HLE cells were compared by wound healing assay; Migration and invasion of these cells were analyzed by Transwell chamber assay; HBx expressed vectors(PcDNA3.1-HBx) were constructed and transfected into L-02 and HLE cells, effects of pcDNA3.1-HBx on the malignant behaviors were also detected by MTT, Transwell chamber assay and the expression of AFP, Ras and CXCR4 were evidenced by Western blotting.

Results: we found that expression of AFP, Ras and CXCR4 in HBV-related HCC and lymph nodes metastasis tissues were significantly elevated compared with HBV-related HCC, non metastasis tissues and HBV-related hepatitis tissues; Expression of AFP, Ras and CXCR4 in HBV-related hepatitis tissues were significantly enhanced compared with normal liver tissues; The growth ratio, migratory and invasive ability, expression of AFP, Ras and CXCR4 of the cells were outstanding promoted while L-02 and HLE cells were transfected with pcDNA3.1-HBx vectors. The proliferation ratio, migration and invasion ability, and expression of Ras and CXCR4 were significantly inhibited while L-02-X and HLE-X cells(stably transfected with pcDNA3.1-HBx) were silenced AFP expression by AFP-siRNA.
\end{abstract}

Conclusions: $\mathrm{HBx}$ through stimulating expression of AFP to promote malignant behaviors of human normal liver cells and HCC cells; AFP maybe used as a novel biotarget for therapeutics of HCC patients.

Key words: Hepatitis B virus X protein(HBx), Alpha fetoprotein(AFP), Hepatocarcinogenesis, Malignant behaviors. 


\section{Introduction}

Chronic infection by hepatitis B virus (HBV) is a major cause of the development and progression of hepatocellular carcinoma (HCC)(1). Among the four viral proteins encoded by an $3.2 \mathrm{~kb} \mathrm{HBV}$ genome, HBV-X (HBx) protein is implicated in hepatocarcinogenesis because it can modulate transcriptional expression of many host cellular genes that are involved in oncogenesis, proliferation, inflammation, and immune responses(2-4). Recent studies have reported that HBx plays an critical role in promoting the invasive and metastatic properties of intrahepatic and extrahepatic metastases of HCC, and poor prognosis in hepatitis B virus-related $\mathrm{HCC}(5-7)$. HBx increases the invasion potential by upregulating the expression of metastasis-associated proteins(8), vascular endothelial growth factors, the membrane-type matrix metalloproteinases(9) and miR-148a(6), which contributes to HCC cells invasion and metastasis. These evidence implicated that $\mathrm{HBx}$ play a role not only in inducing development of HCC, but also in promoting malignant behaviors of HCC cells. Although data indicated that HBx was closely associated with the progression and metastasis of HCC cells, HBx could not directly induce hepatocarcinogenesis, but stimulated the expression of proto-oncogene and other signal molecular proteins to promote HCC occurrence $(10,11)$. These results displayed that transactivation of some HCC occur-associated genes played pivotal role in development and metastasis of HBV-related HCC.

Alpha fetoprotein(AFP) is a specificity biomarker for diagnosing of HCC. Recently, many investigations found that AFP harbors a function to promote malignant transformation of normal liver cells(12-14), and play a role in promoting proliferation and inhibiting apoptosis of HCC cells(15-17), inducing expression of vascular endothelial growth factor(VEGF), VEGF receptor 2, matrix metalloproteinases-2/9 to promote invasion of HCC cells(18). Previously, our studies demonstrated that HBx up-regulated the expression of AFP and AFP receptor priors to other oncogenes, such as Ras, Src in normal liver cells. AFP activated PI3K/AKT signal pathway to promote expression of Ras and $\operatorname{Src}(12,19)$. These results indicate that AFP is a pivotal factor for HBx inducing malignant behaviors of HCC cells. HCC is a major cancer for poor prognosis, because it possesses a character of metastasis and recurrence. Accordingly, in the present study, we investigated the role of $\mathrm{HBx}$ in promoting expression of AFP to stimulate proliferation and metastasis of HCC cells. Further exploration of the mechanisms involved in hepatocarcinogenesis mediated by HBx and AFP. The results displayed that AFP maybe applied as a novel biotarget for the therapeutics of HCC patients.

\section{Material and methods}

\section{Patients and specimens}

The archived clinical specimens were originally collected during hepatectomy of 56 patients, including 15 cases of liver trauma patients (6 case of normal liver specimens, 9 cases of HBV-related hepatitis liver tissues), and 41 cases of HCC specimens (diagnosis confirmed 16 cases non-metastasis and 25 cases metastasis) at Hainan Provincial People's Hospital (Haikou, Hainan, China) and the Affiliated Hospital of the Hainan Medical College (Haikou, Hainan, China) between January 2010 and November 2014. Of the 56 patients, 38 men and 18 women with an average age of 50.8 (range, 31-77) years. All enrolled patients were treated with radical surgery and received no other treatments. Circulating AFP plasma level was measured by enzyme-linked immunosorbent assay(ELISA). Clinical data were obtained by a retrospective chart review. Follow-up was available for all patients. A section of liver tissue about $2.0 \times 2.0 \times 2.0 \mathrm{~cm}$ was obtained from each patient immediately after the surgery. About $1.0 \times 1.0 \times 1.0 \mathrm{~cm}$ tissue samples were fixed in $10 \%$ formalin, embedded in paraffin, and routinely stained with hematoxylin and eosin. The $1.0 \times 1.0 \times 1.0 \mathrm{~cm}$ tissue specimens were stored in liquid nitrogen. All of specimens were assessed blindly and independently by two pathologists. In case of interobserver disagreement, final decisions were achieved by general consensus. All selected patients were diagnosed by histopathological evaluation and metastasis of HCC patients were estimated by computerized tomography (CT). The study protocol was approved by the Ethical Committee of Hainan Provincial People's Hospital and the Science Investigation Ethical Committee of Hainan Medical College. Written informed consent was obtained from all participants.

\section{Immunohistochemical analysis}

The expression and cellular distribution of AFP, Ras and CXCR4 proteins in HCC specimens were assessed by immunohistochemical analysis. Five-millimeter-thick paraffin sections were deparaffinized and re-hydrated according to standard protocols, and heat-induced antigen retrieval was performed in sodium citrate buffer $(10 \mathrm{mmol} / \mathrm{L}, \mathrm{pH}$ 6.0). Endogenous peroxidase was inhibited by $0.3 \%$ $\mathrm{H}_{2} \mathrm{O}_{2}$, and non-specific protein binding was blocked with $10 \%$ goat serum. The sections were then incubated with primary antibody against AFP, Ras 
and CXCR4 (1:100 dilution; Santa Cruz Biotechnology Inc., Santa Cruz, CA, USA) at $4^{\circ} \mathrm{C}$ overnight. Non-immune immunoglobulin G(IgG) was used as a negative control, and antigenic sites were localized using a SP9000 Polymer Detection System and a 3,3'-diaminobenzidine kit (ZSGB-BIO, Beijing, China).

\section{Cell culture}

The non-AFP-producing human HCC cell line (HLE)(20) and human normal liver cell line, L-02 were the gifts from the Department of Cell Biology, Peking University Health Science Center (Beijing, China) and were grown in Dulbecco's modified Eagle's medium(DMED)(Gibco, Carlsbad, CA, USA) supplemented with $10 \%$ fetal calf serum (FCS)(Gibco) and $100 \mathrm{U} / \mathrm{mL}$ penicillin and $100 \mu \mathrm{g} / \mathrm{mL}$ streptomycin. All cell lines were cultured at $37^{\circ} \mathrm{C}$ in a humidified atmosphere with $5 \% \mathrm{CO}_{2}$.

\section{HBx expressed-vectors, AFP expressed-vectors constructed and transfected}

The primers used to create the HBx-expressed vector (pcDNA3.1-HBx) are as follows: Sense 5'-CCGCTCGAGATGGCTGCTAGGCTGTGCTG-3' and Antisense 5'-CGGAATTCTTAGGCAGAGGTGA AAAAGTTG-3'. pcDNA3.1-HBx vectors were transfected into L-02 cells or HLE cells, stably transfected cells are called L-02-X and HLE-X cells. Construction of AFP expressed-vectors(pcDNA3.1-afp) was described as previous(21), and the vectors were transfected into HLE cells. Lipofectamine 2000(Invitrogen, Carlsbad, CA, USA) was applied to promote vectors transfection efficiency.

\section{MTT analysis}

While L-02 cells and HLE cells were transfected with pcDNA3.1-HBx for $24 \mathrm{~h}, 48 \mathrm{~h}$ and $72 \mathrm{~h}$, and L-02-X and HLE-X cells were transfected with AFP-siRNA vectors for $48 \mathrm{~h}$. HLE cells were transfected with pcDNA3.1-afp vectors for $48 \mathrm{~h}$. The growths of these cells were analyzed by MTT methods. The procedure manipulated as previously described(22-24). The cells growth ratio=(treated groups $\left(A_{490}\right)$-control groups $\left.\left(A_{490}\right)\right) /$ control groups $\left(A_{490}\right) \times 100 \%$.

\section{Wound healing assay}

Cells motility was analyzed by a wound healing assay. One day before scratching, HLE and L-02 cells were transfected with pcDNA3.1-HBx vectors and seeded into 12-well plates to almost total confluence in $48 \mathrm{~h}$. A scratching wound was made by scraping the middle of the cell monolayer with a sterile micropipette tip. After all detached cells were washed away with phosphate buffered saline(PBS), the cells were cultured with medium containing 10\% FCS, images of the cells migrating into the wound area were captured at $48 \mathrm{~h}$ by an inverted microscope $(100 \times)$, and their distances were recorded. Cell-repaired motility was evaluated using the following formula: Cell repair ratio $(\%)=$ (non transfected groups distance - transfected groups distance)/ non transfected groups distance $\times 100 \%$.

\section{Cell migration and invasion assay}

Cell migration and invasion assays were carried out according to the manufacturer's protocols. To measure cell migration, transwell chambers were used to observed cultured cells inserts (Transwell chamber; 8-mm pore size; Costar, High Wycombe, UK). The L-02 cells and HLE cells were transfected with pcDNA3.1-HBx vectors for $48 \mathrm{~h}$, and L-02-X cells and HLE-X cells were transfected with AFP-siRNA vectors for $48 \mathrm{~h}$, then the cells were placed into the wells of 12-well cultural plates and the upper and lower chambers were separated. The cells $\left(5 \times 10^{4}\right)$ were added to the upper chamber and cultured with serum-free DMEM medium, whereas the lower chamber was filled with complete medium(contain $20 \%$ FCS). After $48 \mathrm{~h}$ of incubation, the cells in the upper chamber were carefully removed with a cotton swab and those cells that had migrated through the membrane to the lower surface were fixed with $90 \%$ methanol and stained with $0.1 \%$ crystal violet. The number of cells that had migrated through the pores was quantified by counting five independent visual fields under the microscope(Olympus) using a $20 \times$ objective. For invasion assays, transwell chambers were covered with matrigel(BD Falcon, USA), The experimental procedure similar to that for the migration assays. Three independent assays were performed.

\section{Western blotting analysis}

L-02 and HLE cells were transfected with pcDNA3.1-HBx for $24 \mathrm{~h}$ and $48 \mathrm{~h}$, and L-02-X and HLE-X cells were transfected with AFP-siRNA vectors for $48 \mathrm{~h}$, the expression changes of AFP, Ras and CXCR4 in L-02 and HLE cells were evaluated by Western Blotting analysis. Briefly, total proteins were extracted using RIPA lysis buffer (Beyotime Institute

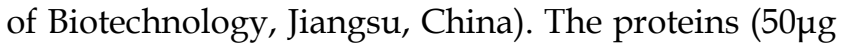
total) were subjected to sodium dodecyl sulfate-polyacrylamide gel electrophoresis and transferred to polyvinylidene fluoride membranes. After incubating with 5\% skim milk in Tris-buffered saline and Tween-20(TBST) at $37^{\circ} \mathrm{C}$ for $30 \mathrm{~min}$, the membranes were probed for the following primary antibodies: mouse anti-AFP(1:500), $\operatorname{Ras}(1: 500)$ or $\beta$-actin(1:1000); rabbit ant-CXCR4(1:500) antibody(all from Santa Cruz Biotechnology Inc.) overnight at $4^{\circ} \mathrm{C}$. After three washes with TBST, the membranes were 
incubated with horseradish peroxidase-conjugated secondary antibodies for $1 \mathrm{~h}$ at $37^{\circ} \mathrm{C}$. The bands were visualized using enhanced chemiluminescence reagents (Thermo Fisher, Rockford, IL, USA) and analyzed with a gel analysis system (VersDoc TM5000MP System; BIO-RAD, Guangzhou, China). The expression of $\beta$-actin was used as loading control.

\section{Statistical analysis}

The results of multiple observations were presented as mean \pm sd of at least three independent experiments. Statistical significance was determined using Student's t-test and One-way ANOVA(SPSS 11.5 software for Windows, SPSS Inc., Chicago, IL, US).

\section{Results}

\section{Expression of AFP, Ras and CXCR4 positively correlated with metastasis of HBV-related HCC cells.}

In this study, we analyzed the expression of AFP, Ras and CXCR4 in clinical patients' liver tissue samples by immunohistochmeistry staining. The results indicated that expression of AFP, Ras and CXCR4 gradually increased in normal liver tissue $\rightarrow$ hepatitis tissue $\rightarrow$ non metastasis HCC tissue $(\mathrm{HBV}+) \rightarrow$ metastasis $\mathrm{HCC}$ tissue $(\mathrm{HBV}+$ ) (Figure 1A). Serum concentration of AFP was detected by ELISA in these participators, the results showed that AFP concentration elevated in metastasis HCC patients than that in non metastasis HCC patients and other non cancer participants (Figure 1B). Western blotting detection also displayed expression of AFP, Ras and CXCR4 significantly up-regulation in metastatic HCC patients than that in non metastasis HCC patients and other non cancer participants (Figure 1C). These data implicated that high expression of AFP, Ras and CXCR4 was positively associated with metastasis of HBV-related HCC cells.

\section{HBx promoted proliferation of normal liver cells and HCC cells}

In order to explore the effect of $\mathrm{HBx}$ on the proliferation of normal liver cells and HCC cells, L-02 cells and HLE cells were transfected with HBx-expressed vectors(pcDNA3.1- $H B x$ ). MTT method was used to analyze the growth of the cells. In this investigation, we found that the high transfection efficiency of pcDNA3.1-HBx vectors in L-02 cells and HLE cells (Figure 2A), this result proved that L-02 cells and HLE cells carried $H B x$ gene. MTT analysis indicated that the proliferation of L-02 cells and HLE cells were significantly promoted while transfected with pcDNA3.1-HBx vectors for $24 \mathrm{~h}, 48 \mathrm{~h}$ and $72 \mathrm{~h}$, the growth incremental ratio of L-02 cells were
(74.5 \pm 7.6$) \%,(39.1 \pm 3.4) \%$ and $(91.3 \pm 8.9) \%$ respectively, and the growth incremental ratio of HLE cells were $(60.9 \pm 5.3) \%,(40.2 \pm 3.8) \%$ and $(51.9 \pm 4.6) \%$ respectively (Figure 2B).The statistical analysis significance p-value is less than $0.05 \quad(P<0.05)$ showing the transfected with pcDNA3.1-HBx vectors vs control and vehicle pcDNA3.1 vectors. These results indicated that $\mathrm{HBx}$ played a role in promoting the growth of normal liver cells and HCC cells.

\section{HBx promoted normal liver cells and HCC cells scarify healing, migration and invasion in vitro}

Using wound healing assay, we found that while L-02 cells and HLE cells were transfected with HBx-expressed vectors, pcDNA3.1- $H B x$, for $48 \mathrm{~h}$, the scarify healing ratio significantly increased compared to control group and vehicle pcDNA3.1 vectors group, the healing ratio of L-02 cells were $17.72 \pm 3.61$ (control groups) and 16.05 \pm 2.93 (vehicle pcDNA3.1 vectors groups) vs $69.25 \pm 6.84$ (pcDNA3.1- $H B x$ groups), $P<0.01$; The healing ratio of HLE cells were 18.37 \pm 5.08 (control groups) and $19.10 \pm 5.08$ (vehicle pcDNA3.1 vectors groups) vs $76.95 \pm 8.04$ (pcDNA3.1-HBx groups), $P<0.01$ (Figure $3 \mathrm{~A})$. In order to further assess the influence of pcDNA3.1-HBx vectors on L-02 and HLE cells migration and invasion, we employed cell transwell migration and invasion assays to determine effects of HBx on malignant progression and metastasis. The results showed that numbers of migratory L-02 cells which transfected with pcDNA3.1-HBx vectors were significantly higher compared to the control groups and vehicle pcDNA3.1 vectors groups after transfected for $48 \mathrm{~h}(20.80 \pm 4.96$ and $20.40 \pm 8.61$ vs $49.80 \pm 7.08, P<0.01)$. Similarly, numbers of migratory HLE cells which transfected with pcDNA3.1-HBx vectors were significantly higher compared to the control groups and vehicle pcDNA3.1 vectors groups after transfected for $48 \mathrm{~h}(26.20 \pm 7.05$ and $28.00 \pm 7.09 \mathrm{vs}$ $59.80 \pm 10.94, P<0.05$ ) (Figure $3 \mathrm{~B}$ ). The similar results were also observed in invasion assay, numbers of L-02 and HLE which carried pcDNA3.1-HBx vectors were a significantly higher compared to control group and vehicle pcDNA3.1 vectors groups after transfected for $48 \mathrm{~h}$, number of invasive L-02 cells were $17.40 \pm 5.94$ (control groups) and 18.40 \pm 3.57 (vehicle pcDNA3.1 vectors groups) vs $47.20 \pm 8.75$ (pcDNA3.1- $H B x$ groups), $P<0.05$ and numbers of HLE cells were $14.20 \pm 4.81$ (control groups) and $15.60 \pm 5.94$ (vehicle pcDNA3.1 groups) vs $37.40 \pm 8.84$ (pcDNA3.1-HBx groups), $P<0.05$ (Figure 3C); These results displayed an enhanced effect of HBx on L-02 and HLE cells migration and invasion. 
A

HE stained

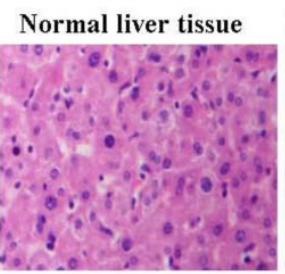

Hepatitis tissue(HBV+)

HCC tissue(HBV+)
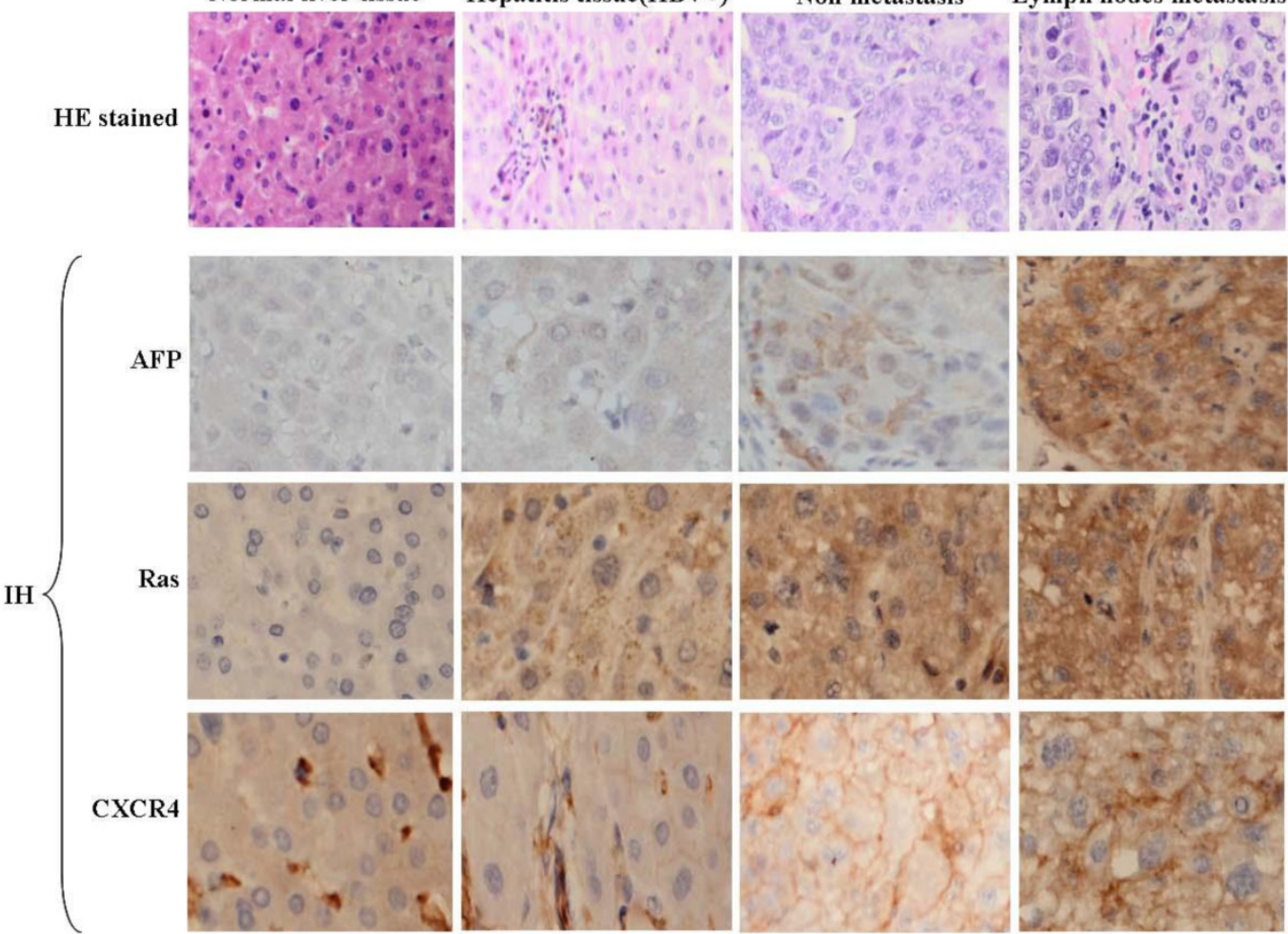

B

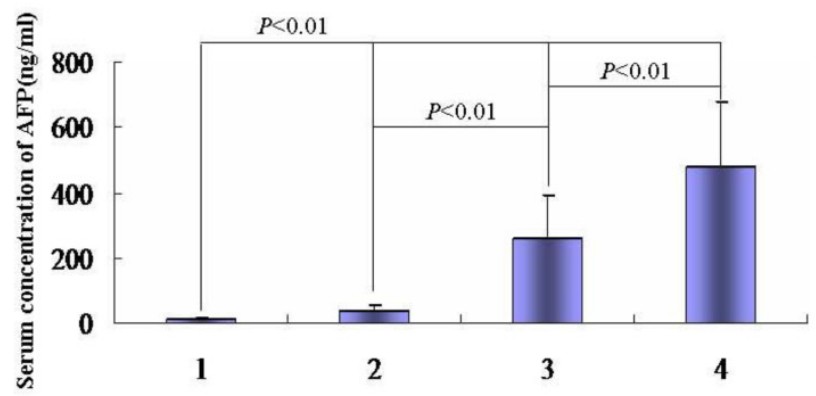

$\mathbf{C}$
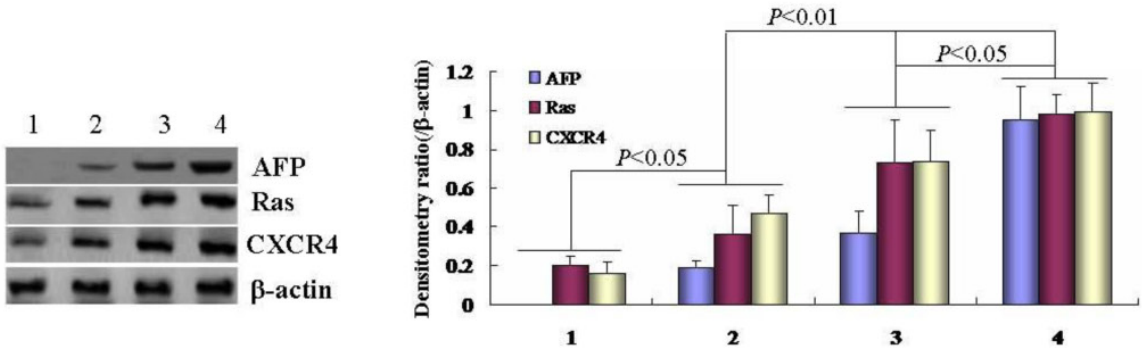

Figure 1. Correlation of HBV with expression of AFP, Ras and CXCR4 in HCC tissues. Clinical liver tissues sample were collected after surgical hepatectomy. A, Expression of AFP, Ras and CXCR4 in the tissues were detected by immunohistochemistry assay. B, Concentration of serum AFP was detected by ELISA. 1, Normal; 2, Hepatitis patients(HBV+); 3, Non metastasis HCC patients(HBV+); 4, Lymph nodes metastasis HCC patients(HBV+). C, Expression of AFP, Ras and CXCR4 in the tissues were detected by Western blotting, right column images represented the proteins densitometry value ratio compared with internal control $\beta$-actin. 1 , Normal liver tissues; 2 , Hepatitis tissues(HBV+); 3, Non metastasis $\mathrm{HCC}$ tissues $(\mathrm{HBV}+)$; 4, Lymph nodes metastasis $\mathrm{HCC}$ tissues $(\mathrm{HBV}+)$. The images representation of at last three reduplicate experiments. $\mathrm{HE}$ stained: Haematoxylin and eosin stained; IH: Immunohistochmeistry stained. 

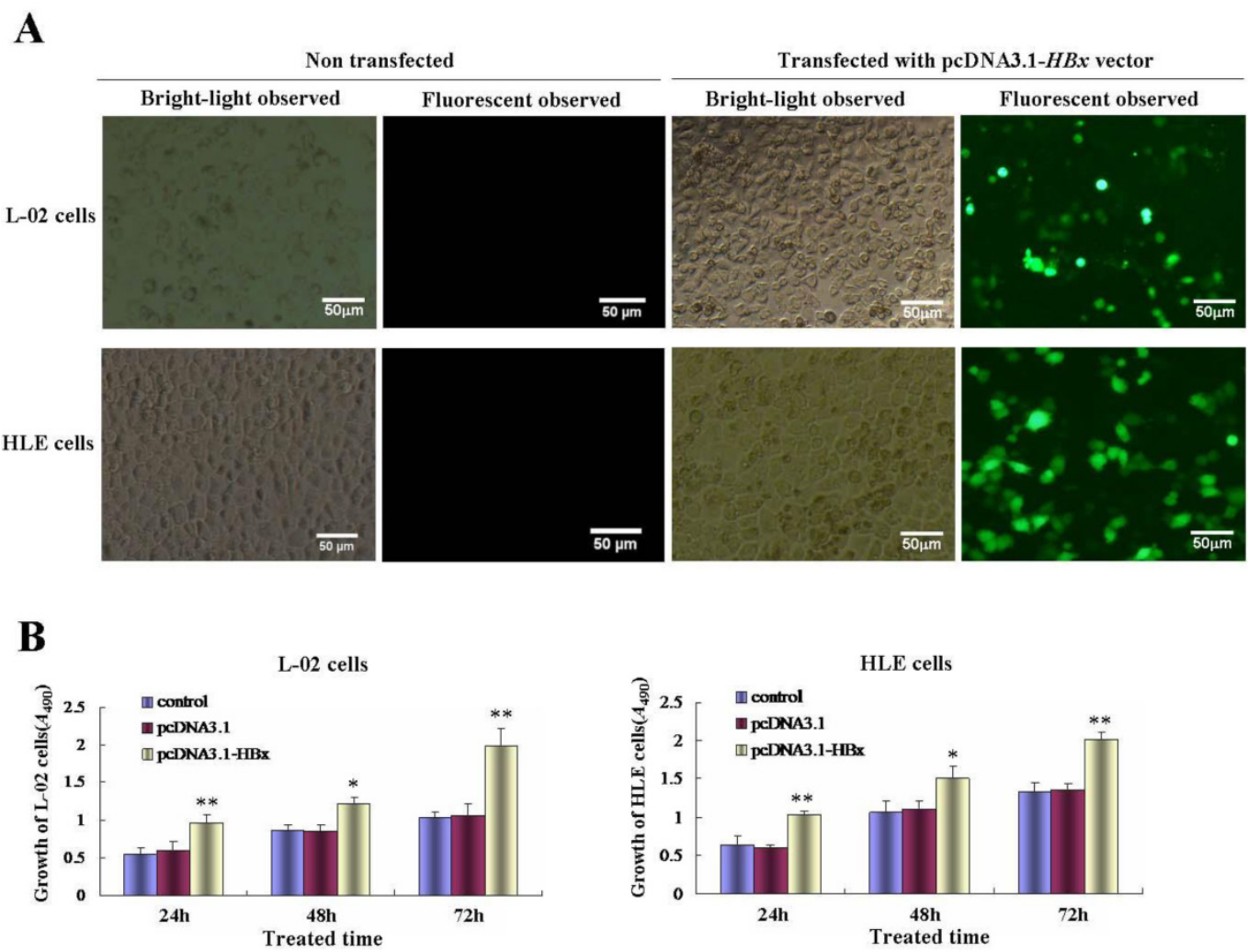

Figure 2. Effects of $\mathbf{H B x}$ on the growth of human normal liver cells line L-02 and human hepatoma cell line HLE. A, Efficiency of pcDNA3.1-HBx vectors were transfected into human normal liver cells line, L-02 cells, and human hepatoma cells line, $\mathrm{HLE}$ cells. L- 02 cells and $\mathrm{HLE}$ cells were cultured in $95 \% \mathrm{O}_{2} / 5 \% \mathrm{CO}_{2}$ and humid environment, fluorescent microscopy were applied to observe the transfected efficient while L-02 cells and HLE cells were transfected with pcDNA3.1-HBx vectors for 48 hours. The images were representation of three independent experiments. B, L-02 cells and HLE cells were transfected with pcDNA3.1-HBx vectors for 24,48 and 72 hours respectively, the growth of $L-02$ cells and HLE cells were detected by MTT methods. ${ }^{*} P<0.05$ vs control and $p c D N A-3.1$ groups; $* * P<0.01$ vs control and $p c D N A-3.1$ groups. We carried out at last three reduplicate experiments.

\section{HBx promoted expression of AFP, Ras and CXCR4 in normal liver cells and HCC cells}

The growth and metastasis of HCC cells were involved in a high expression of AFP, Ras and CXCR4(25-28). In the present study, we evaluated the influence of $\mathrm{HBx}$ on expression of AFP, Ras and CXCR4 in L-02 cells and HLE cells. Western blotting analyzed indicated that there were significantly up-regulated expressions of AFP, Ras and CXCR4 in L-02 cells and HLE cells while transfected with pcDNA3.1- $H B x$ vectors for $24 \mathrm{~h}$ or $48 \mathrm{~h}$ (Figure 4 ). These results suggested that $\mathrm{HBx}$ enhanced proliferation and metastasis of L-02 cells and HLE cells maybe through stimulating expression of AFP, Ras and CXCR4.

\section{Silenced expression of AFP inhibited HBx promoted expression of Ras and CXCR4}

L-02 cells and HLE cells were stably transfected with pcDNA3.1-HBx vectors, named L-02-X and HLE-X respectively. Previously, the results had demonstrated that L-02-X and HLE-X expressed AFP, Ras and CXCR4 (Figure 4). In order to explore the mechanism of HBx induced malignant behaviors of L-02 cells and HLE cells, in this investigation, we silenced AFP expressed by siRNA in L-02-X cells and HLE-X cells. The results showed that suppressed expression of AFP, led to the expression of Ras and CXCR4 were significantly inhibited in these cells (Figure 5). The results proved that HBx induced expression of AFP was a pivotal factor for stimulating expression of Ras and CXCR4 in L-02 cells and HLE cells.

\section{Silence expression of AFP restrained growth and metastasis of L-02 cells and HLE cells induced by HBx; And AFP promoted proliferation of HLE cells}

In order to evaluate the effect of AFP on the HBx mediated the growth and metastasis of L-02-X cells and HLE cells, we silenced AFP expression by siRNA. MTT assay indicated that the proliferation of L-02-X cells and HLE-X cells were significantly suppressed while these cells were transfected with AFP-siRNA vectors at $48 \mathrm{~h}$, the growth restrained ratio was $(50.62 \pm 8.96) \%$ of L-02-X cells, and the growth restrained ratio was $(57.18 \pm 9.32) \%$ of HLE-X cells, statistic analysis showed that $P<0.01$ compared to control group and scramble-siRNA vectors groups (Figure 6A). 


\section{A}
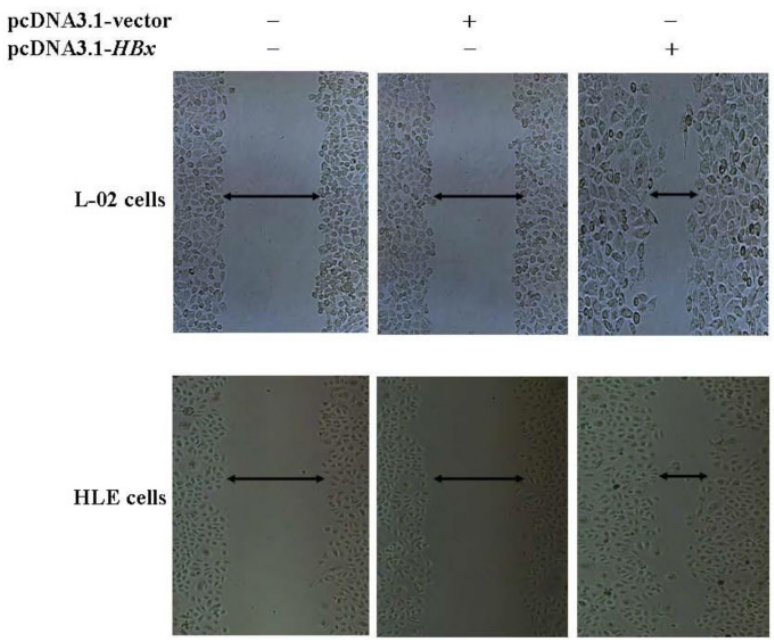

\section{B}

pcDNA3.1-vector pcDNA3.1- $H B x$

L-02 cells
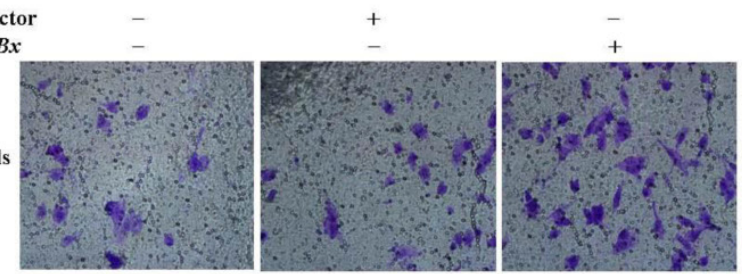

pcDNA3.1-vec pcDNA3.1-HB
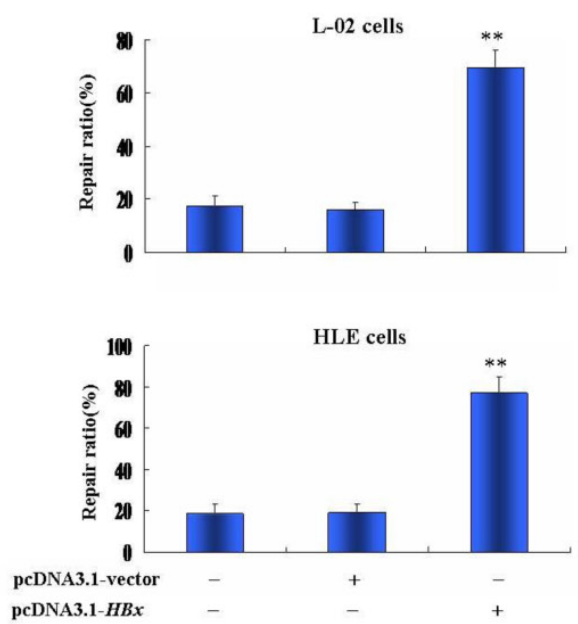
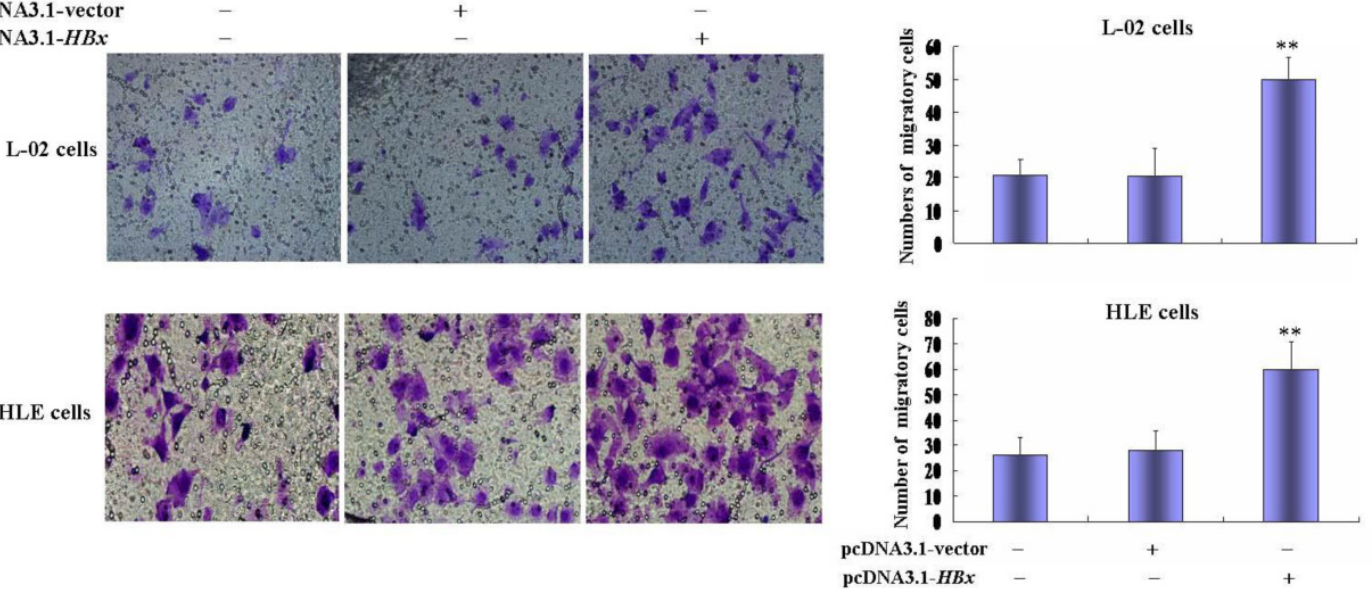

\section{C}

pcDNA3.1-vector pcDNA3.1- $H B$ x
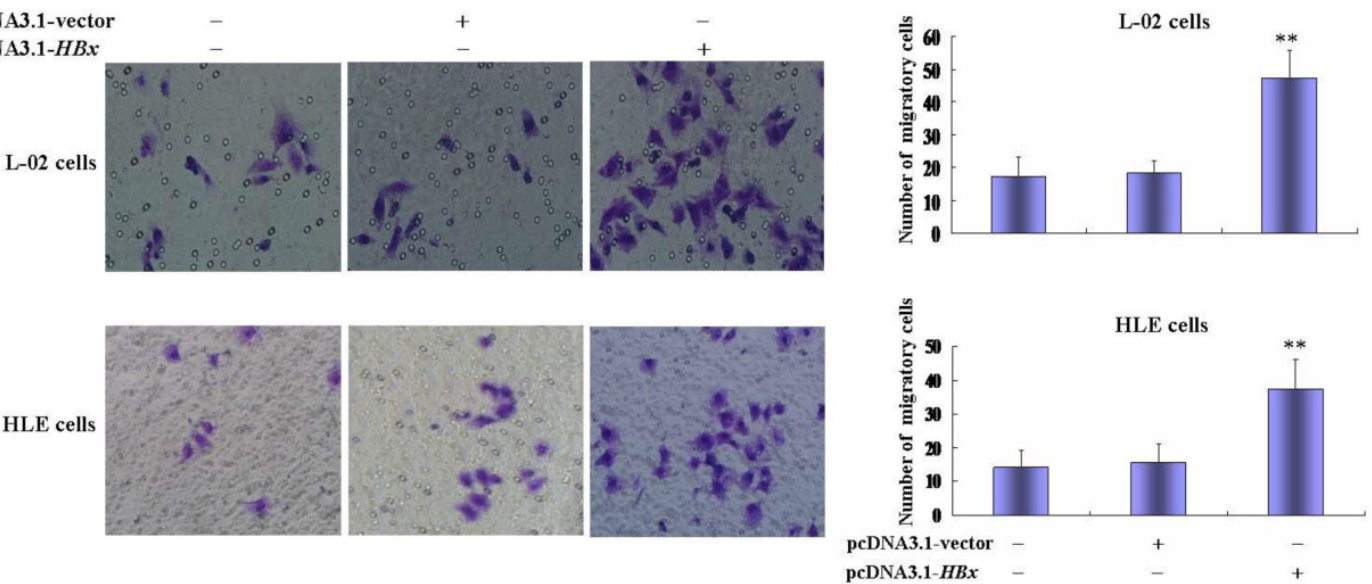

Figure 3. Effects of HBx on the malignant behaviors of L-02 cells and HLE cells. A, L- 02 cells and HLE cells were transfected with pcDNA3.1-HBx for 48 hours, migratory ability of L- 02 cells and HLE cells were compared by wound healing assay, wound closure was observed by microscopy. Right column image represent the heal ratio of these cells. **P<0.01. vs non treated groups and pcDNA3.1 vectors groups. B, Effects of $\mathrm{HBx}$ on migratory ability of L-02 cells and HLE cells were compared by Transwell migration assay. L- 02 cells and HLE cells were transfected with pcDNA3.1-HBx for 48 hours. The numbers of migratory cells were calculated by microscopy. Right column image represent the numbers of these cells. $* * P<0.01$ vs non treated groups and pcDNA3.1 vectors groups. C, Effects of HBx on invasive ability of L-02 cells and HLE cells were compared by Transwell invasion assay. L- 02 cells and HLE cells were transfected with pcDNA3.1-HBx for 48 hours. The numbers of invasive cells were calculated by microscopy. Right column image represent the numbers of these cells. $* * P<0.01$ vs non treated groups and $p c D N A 3.1$ vectors groups. We carried out at last three reduplicate experiments. 

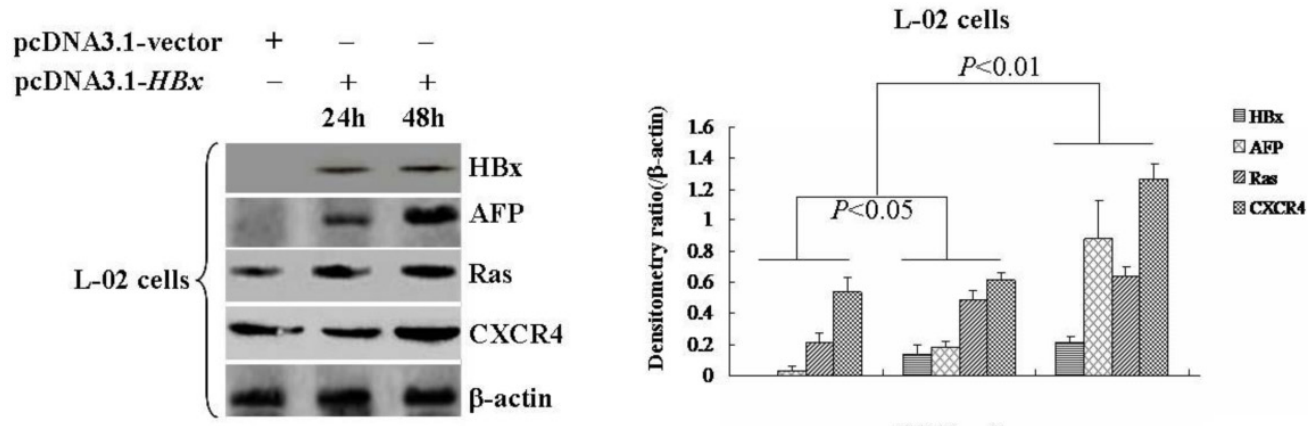

HLE cells
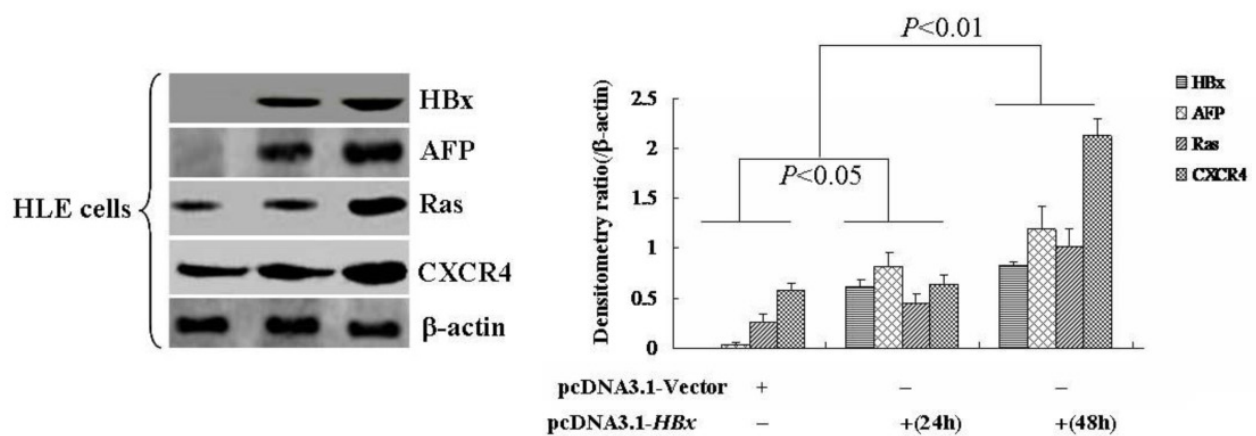

Figure 4. Effects of HBx on the expression of AFP, Ras and CXCR4 in L-02 cells and HLE cells. L-02 cells and HLE cells were transfected with pCDNA3.1-HBx for 24 hours or 48 hours. Expression of AFP, Ras and CXCR4 were analyzed by Western blotting. Right column images represented protein densitometry value ratio compared to $\beta$-actin. The images representation of at last three reduplicate experiments.

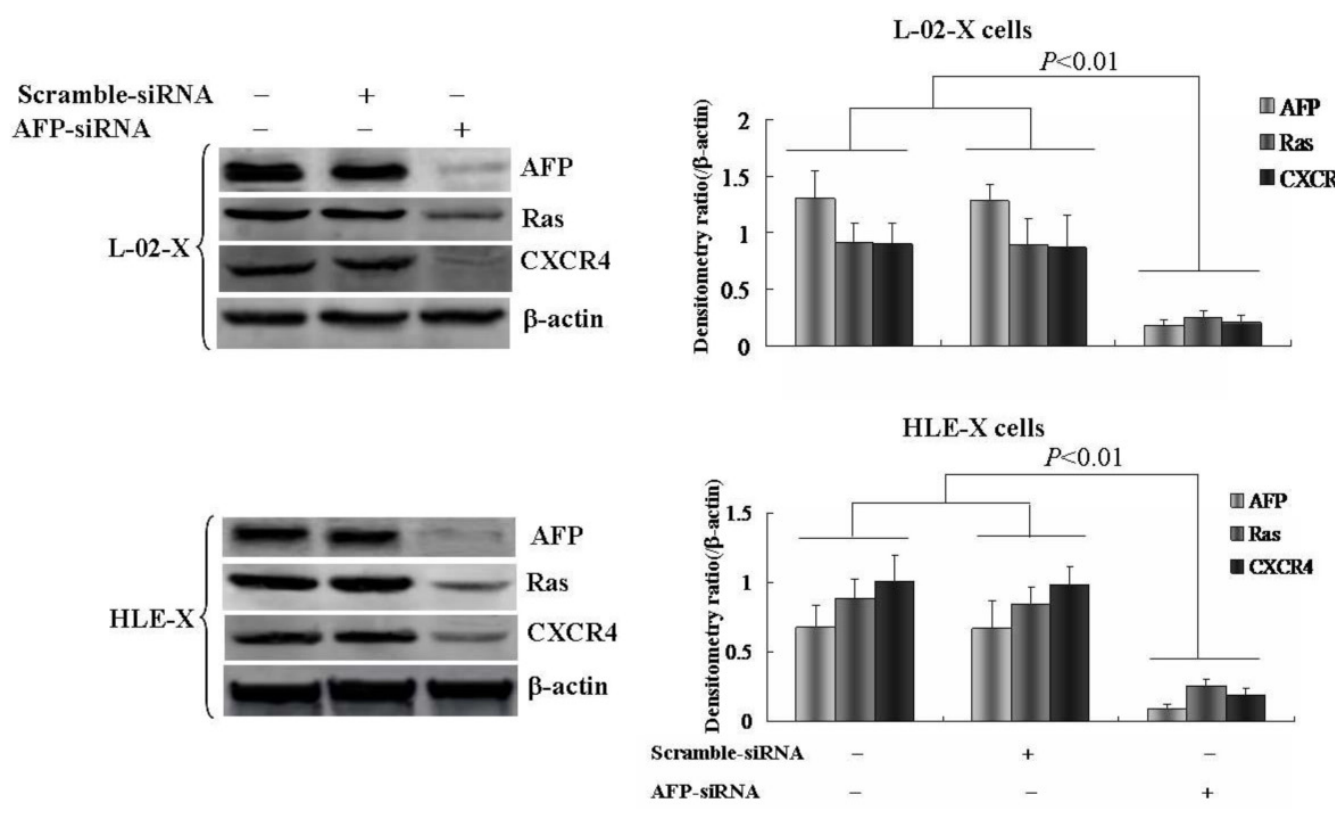

Figure 5. Effects of AFP on the expression of Ras and CXCR4 in L-02-X cells and HLE-X cells (Stable transfected with pcDNA3.1-HBX vectors). L-02-X cells and HLE-X cells were transfected with AFP-siRNA for 48 hours, expresssion of Ras and CXCR4 were detected by Western blotting. Right column images represented densitometry value ratio compared to $\beta$-actin. $* * P<0.01$ vs non treated groups and scramble-siRNA groups. The images representation of at last three reduplicate experiments. 
A

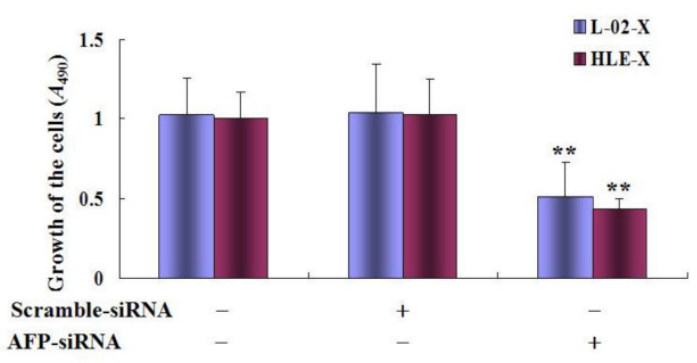

B

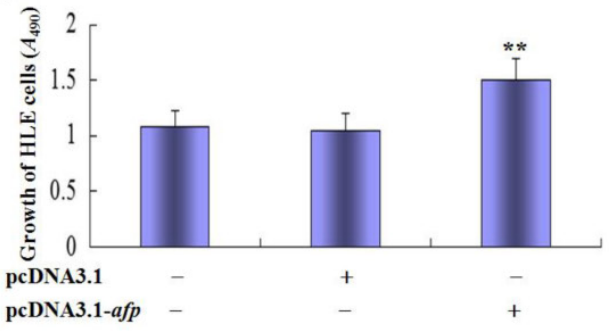

C

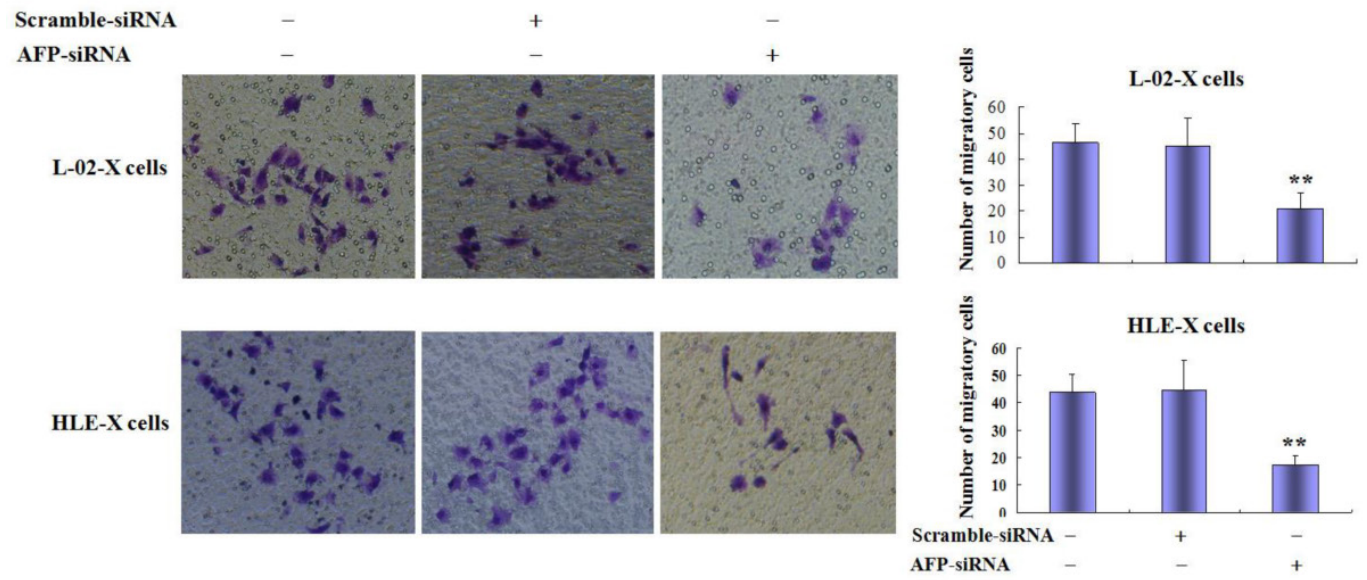

D

Scramble-siRNA

AFP-siRNA
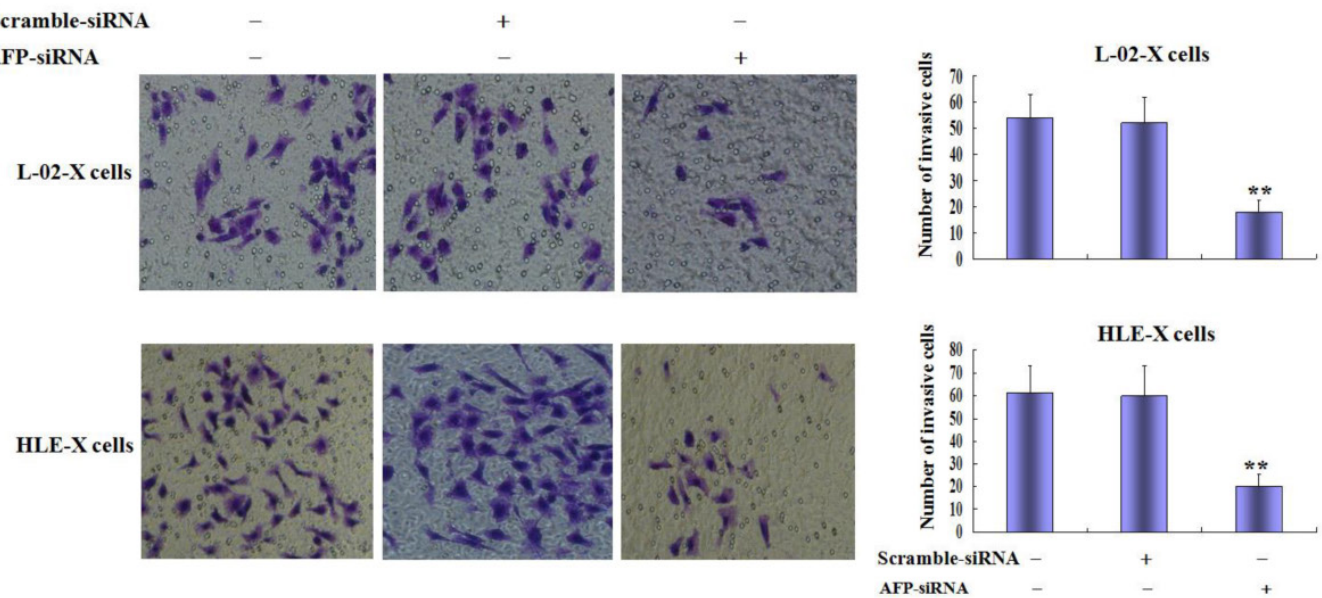

Figure 6. Effects of AFP on malignant behaviors of L-02-X cells and HLE-X cells, and proliferation of HLE cells. A, L-02-X cells or HLE-X cells were transfected with AFP-siRNA for 48 hours, growth of these cells were analyzed by MTT method, $* * P<0.01$ vs non treated groups and scramble-siRNA groups. B, HLE cells were transfected with overexpresion vectors(pcDNA3.1-afp) for 48 hours, the proliferation of HLE cells were detected by MTT; **P<0.01 vs non treated groups and pcDNA3.1 vectors groups. C, L-02-X cells or HLE-X cells were transfected with AFP-siRNA for 48 hours, the migration of L-02-X cells or HLE-X cells were assayed by Transwell, numbers of migratory cells were calculated by microscopy, right column image represent the numbers of these cells. $* * P<0.01$ vs non treated groups and scramble-siRNA groups. $D$, L-02-X cells or HLE-X cells were transfected with AFP-siRNA for 48 hours, the invasion of L-02-X cells or HLE-X cells were assayed by Transwell, numbers of invasive cells were calculated by microscopy, right column image represent the numbers of these cells. $* * P<0.01$ vs non treated groups and scramble-siRNA groups. The images representation of at last three reduplicate experiments.

In the present study, we also observed the role of AFP in regulating the growth of HLE cells, the results indicated that the growth ratio was significantly higher while HLE cells were transfected with pcDNA3.1-afp vectors compared to non treated groups and transfected with pcDNA3.1 vectors groups(Figure 6B). Transwell chamber migration assay displayed that the numbers of migratory L-02-X cells were significantly decreased while transfected with AFP-siRNA vectors for $48 \mathrm{~h}$, numbers of migratory cells of control groups, scramble-siRNA vectors groups and AFP-siRNA were 46.5 \pm 7.32 , $45.0 \pm 10.8$ and 21.0 \pm 5.9 respectively; Similarly, HLE-X cells were transfected with AFP-siRNA vectors for 
$48 \mathrm{~h}$, the numbers of migratory cells were significantly decreased, numbers of migratory cells of control groups, scramble-siRNA vectors group and AFP-siRNA groups were $43.7 \pm 6.6,44.7 \pm 10.6$ and 17.2 \pm 3.5 respectively (Figure $6 \mathrm{C}$ ). Transwell chamber invasion assay also indicated that silenced expression of AFP significantly decreased invasive cells numbers of L-02-X cells and HLE- $X$ cells, the L-02-X invasive cells numbers of control groups, scramble-siRNA vectors groups and AFP-siRNA groups were 54.0 \pm 9.0 , $51.8 \pm 9.8$ and $18.2 \pm 4.9$ respectively; Similarly, the HLE-X invasive cells numbers of control group, scramble-siRNA vectors groups and AFP-siRNA groups were 54.0 $\pm 9.0, \quad 51.8 \pm 9.8$ and 18.2 \pm 4.9 respectively (Figure 6D). These results implicated that AFP harbors a trait to stimulate growth of HLE cells, and AFP played a critical role in $\mathrm{HBx}$ promoting malignant behaviors of normal liver cells and HCC cells.

\section{Discussion}

HCC is one of the most common cancers in the world, and approximately $90 \%$ of the HCC cases are associated with HBV infection. It has been reported that HBx is a key factor in hepatocarcinogenesis(4), but HBx itself is a poor transformer of human and rodent hepatic cells. Indeed, $\mathrm{HBx}$ induced hepatocarcinogenesis through activating growth signal pathway, such as STATs and PI3K/AKT signal(19,29-31), activation of these signal pathway promote expression of Src, Ras and matrix metalloproteinase 2/9(13,32); HBx also inhibited transcripted activity of p53 to suppress expression of PTEN(33-35), and promote expression of $\operatorname{AFP}(36,37)$;

Recently, some new evidences indicated that $\mathrm{HBx}$ regulated expression of microRNA, such as stimulated expression of miRNA-21(38) and restrained expression of miRNA-148a to promote early carcinogenesis of hepatocytes(39). These data further supported that expression of oncogenes and cancer suppressors played important role of $\mathrm{HBx}$ in driving malignant transformation of liver cells.

In this study, we found that expression of AFP, Ras and CXCR4 positively correlated with the progression of HBV-related HCC. Interestingly, high serum concentration of AFP associated to metastasis of HCC patients, these results revealed that expression of AFP, Ras and CXCR4 played an important role in HBx promoting development and metastasis of HCC cells in vivo. In order to explore the role of HBx in the malignant transformation of liver cells and malignant behaviors of HCC cells, we constructed HBx-expressed vectors (pcDNA3.1-HBx) and transfected into normal liver cells, L-02 and HCC cells, HLE. MTT assay showed that HBx-expressed vectors accelerated growth not only in L-02 cells, but also in HLE cells. Previously, documents have proved that HBx promoted proliferation of L-02 cells $(40,41)$, although data indicated that HBx perhaps inhibit p53 activity in HLE cells(42), the role of HBx in promoting growth of HLE cells is still unclear. In the present study, we found that HBx was able to promote growth of HLE cells. These results demonstrated that $\mathrm{HBx}$ played a role in stimulating proliferation of normal liver cells and HCC cells.

Migration and invasion were important characteristic of malignant behaviors of cancer cells. In the present study, we analyzed the effect of HBx on the migration and invasion of L-02 and HLE cells. The wound healing assay indicated that $\mathrm{HBx}$ healed the scarification of L-02 cells and HLE cells. The Transwell chamber assay also demonstrated that while the L-02 cells and HLE cells were transfected with $\mathrm{HBx}$-expressed vectors (pcDNA3.1-HBx), the migration and invasion of the cells were significantly promoted. Further investigation, the results indicated that HBx was able to stimulate expression of AFP, Ras and CXCR4 in L-02 cells and HLE cells. Previous studies have proved that the migration and invasion of HCC cells involve in overexpression of Ras and CXCR4(43-45), we had found that HBx priors to induce expression of AFP to promote expression of Ras and CXCR4 in normal liver cells and HCC cells(13), and AFP mediated HBx driving malignant transformation of normal liver cells(46). In the present investigation, the results elucidated that $\mathrm{HBx}$ induced expression of AFP, Ras and CXCR4 to stimulate wound healing, migration and invasion of L-02 cells and HLE cells.

AFP was an early biomarker for monitoring HCC development, many studies showed that AFP harbors a function to promote proliferation of normal liver cells and HCC cells(14,47-50). HBx stimulates malignant transformation of L-02 cells via enhancing expression of $\mathrm{AFP}(14)$, we previous study indicated that $\mathrm{HBx}$ driven expression of AFP to promote expression of Src and CXCR4(13). In the present investigation, in order to explore the mechanism of AFP in mediating HBx promoted malignant behaviors of L-02 cells and HLE cells, we silenced AFP gene by siRNA in L-02-X cells and HLE-X cells which stable trsanfected with HBx-expressed vectors. The results indicated that growths of these cells were significantly suppressed, and the capacity of migration and invasion were also restrained. Western Blotting assay displayed that inhibited AFP expression led to the expression of Ras and CXCR4 were repressed, MTT results also indicated that AFP was able to stimulate growth of HLE cells. These results implicated that AFP was a critical factor for mediating $\mathrm{HBx}$ promoted 
malignant behaviors of normal liver cells and HCC cells, HBx driven AFP expression was an important step for inducing HCC occurrence and malignant behaviors of cancer cells. Recently, we had found that AFP activated PI3K/AKT/mTOR signal pathway to promote expression of Ras, Src and CXCR4 $(13,19,24,25)$, because Ras, Src and CXCR4 played important role in the growth and metastasis of HCC cells(51-53), the present results suggested that HBx stimulated malignant behaviors of L-02 cells and HLE cells through driving expression of AFP. We speculated that AFP is a pioneer factor for $\mathrm{HBx}$ inducing hepatocarcinogenesis, AFP maybe was applied as a novel biotarget molecule for the therapeutics of HCC patients.

Altogether, this study is the first time to demonstrate that AFP was a pivotal molecule for $\mathrm{HBx}$ driving malignant behaviors of normal liver cells and HCC cells. HBx plays its functions through stimulating AFP expression to promote expression of oncogene, such as Ras, Src, and metastasis-related gene, CXCR4. These effects perhaps explain why HBx appears prior to drive AFP expression in early malignant transformation of liver cells. AFP is applied not only for monitoring hepatocarcinogenesis, but also has exerted as a novel biotarget molecule for HCC therapy.

\section{Abbreviations}

AFP: alpha fetoprotein; HBx: Hepatitis B virus $X$ protein; HCC: hepatocellualr carcinoma; CXCR4: CXC chemokine receptors 4; PI3K/AKT: phosphatidylinositol 3-kinase/protein kinase B; MTT: 3-(4,5-dimethylthiazol-2-yl)-2,5-diphenyltetrazolium bromide.

\section{Supplementary Material}

Supplementary table.

http://www.jcancer.org/v07p0935s1.pdf

\section{Acknowledgements}

This work was supported by the National Natural Science Foundation of China (Nos. 81560450, 31560243, 81360307, 81260306, 81160261, 31060164 and 30960153); Project of Hainan Province Innovative Team(No. 2016CXTD008); Key Projects of Science and Technology, Hainan Province (No. ZDXM 20110038); Natural Science Foundation of Hainan Province (Nos. 309034, 310044 and 814293); and Fund of Hainan Provincial Society Development (No. 2015SF03); Hainan Provincial Department of Education (No. HjKj 2013-21); Hainan Provincial Association for Science and Technology program of Youth Science Talent and Academic Innovation(No.201514); Grants from the Hnkyzx2014-07, and Haikou Key Program of Science \& Technology (No. 2014062).

\section{Competing Interests}

The authors have declared that no competing interest exists.

\section{References}

1. Neuveut C, Wei Y, Buendia MA. Mechanisms of HBV-related hepatocarcinogenesis. J Hepatol. 2010; 52(4):594-604.

2. Zhang $\mathrm{W}$, Lu Z, Kong G, et al. Hepatitis $B$ virus $X$ protein accelerates hepatocarcinogenesis with partner survivin through modulating miR-520b and HBXIP. Mol Cancer. 2014;13:128.

3. Zhang T, Zhang J, Cui M, et al. Hepatitis B virus $X$ protein inhibits tumor suppressor miR-205 through inducing hypermethylation of miR-205 promoter to enhance carcinogenesis. Neoplasia. 2013;15(11):1282-1291.

4. Zhang $X$, Zhang $H$, Ye L. Effects of hepatitis $B$ virus $X$ protein on the development of liver cancer. J Lab Clin Med. 2006; 147(2):58-66.

5. Huang JF, Guo YJ, Zhao CX, et al. Hepatitis B virus X protein (HBx)-related long noncoding RNA (lncRNA) down-regulated expression by HBx (Dreh) inhibits hepatocellular carcinoma metastasis by targeting the intermediate filament protein vimentin. Hepatology. 2013;57(5):1882-1892.

6. Xu X, Fan Z, Kang L, et al. Hepatitis B virus X protein represses miRNA-148a to enhance tumorigenesis. J Clin Invest. 2013;123 (2):630-645.

7. Xia L, Huang W, Tian D, et al. Upregulated FoxM1 expression induced by hepatitis $B$ virus $X$ protein promotes tumor metastasis and indicates poor prognosis in hepatitis B virus-related hepatocellular carcinoma. J Hepatol. 2012;57(3):600-612.

8. Lee MH, Na H, Na TY, Shin YK, Seong JK, Lee MO. Epigenetic control of metastasis-associated protein 1 gene expression by hepatitis $B$ virus $X$ protein during hepatocarcinogenesis. Oncogenesis. 2012;1e25.

9. Liu LP, Liang HF, Chen XP, et al. The role of NF-kappaB in Hepatitis b virus $X$ protein-mediated upregulation of VEGF and MMPs. Cancer Invest. 2010; 28(5):443-451.

10. Twu JS, Lai MY, Chen DS, Robinson WS. Activation of protooncogene c-jun by the X protein of hepatitis B virus. Virology. 1993;192(1):346-350.

11. Caselmann WH. Transactivation of cellular gene expression by hepatitis $B$ viral proteins: a possible molecular mechanism of hepatocarcinogenesis. J Hepatol. 1995;22(1 Suppl):34-37.

12. Li M, Zhu M, Li W, et al. Alpha-fetoprotein receptor as an early indicator of $\mathrm{HBx}$-driven hepatocarcinogenesis and its applications in tracing cancer cell metastasis. Cancer Lett. 2013;330(2):170-180.

13. Zhu M, Guo J, Li W, et al. Hepatitis B virus $X$ protein induces expression of alpha-fetoprotein and activates PI3K/mTOR signaling pathway in liver cells. Oncotarget. 2015; 6(14): 12196-12208.

14. Zhang WY, Cai N, Ye LH, Zhang XD. Transformation of human liver L-O2 cells mediated by stable $\mathrm{HBx}$ transfection. Acta Pharmacol Sin. 2009;30(8):1153-1161.

15. Gao R, Cai C, Gan J, et al. miR-1236 down-regulates alpha-fetoprotein, thus causing PTEN accumulation, which inhibits the PI3K/Akt pathway and malignant phenotype in hepatoma cells. Oncotarget. 2015;6(8):6014-6028.

16. Yang X, Zhang Y, Zhang L, Zhang L, Mao J. Silencing alpha-fetoprotein expression induces growth arrest and apoptosis in human hepatocellular cancer cell. Cancer Lett. 2008;271(2):281- 293.

17. Zheng L, Gong W, Liang P, et al. Effects of AFP-activated PI3K/Akt signaling pathway on cell proliferation of liver cancer. Tumour Biol. 2014; 35(5):4095-4099.

18. Meng W, Li X, Bai Z, et al. Silencing alpha-fetoprotein inhibits VEGF and MMP-2/9 production in human hepatocellular carcinoma cell. PLoS One. 2014;9(2):e90660

19. Zhu M, Guo J, Li W, et al. HBx induced AFP receptor expressed to activate $\mathrm{PI} 3 \mathrm{~K} / \mathrm{AKT}$ signal to promote expression of Src in liver cells and hepatoma cells. BMC Cancer. 2015;15:362.

20. Yokoo H, Kondo T, Fujii K, Yamada T, Todo S, Hirohashi S. Proteomic signature corresponding to alpha fetoprotein expression in liver cancer cells. Hepatology. 2004; 40(3): 609- 617.

21. $\mathrm{Li} \mathrm{M}, \mathrm{Li} \mathrm{H}, \mathrm{Li} \mathrm{C}$, et al. Cytoplasmic alpha-fetoprotein functions as a co-repressor in RA-RAR signaling to promote the growth of human hepatoma Bel7402 cells. Cancer Lett. 2009; 285(2): 190-199.

22. Li MS, Li PF, Yang FY, He SP, Du GG, Li G. The intracellular mechanism of alpha-fetoprotein promoting the proliferation of NIH 3T3 cells. Cell Res. 2002;12(2):151-156.

23. Li MS, Li PF, He SP, Du GG, Li G. The promoting molecular mechanism of alpha-fetoprotein on the growth of human hepatoma Bel7402 cell line. World J Gastroenterol. 2002;8(3):469-475.

24. $\mathrm{Li} \mathrm{M}, \mathrm{Li} \mathrm{H}, \mathrm{Li} \mathrm{C}$, et al. Alpha-fetoprotein: a new member of intracellular signal molecules in regulation of the PI3K/AKT signaling in human hepatoma cell lines. Int J Cancer. 2011;128(3):524-532. 
25. Zhu M, Guo J, Xia H, et al. Alpha-fetoprotein activates AKT/mTOR signaling to promote CXCR4 expression and migration of hepatoma cells. Oncoscience. 2015;2(1):59-70.

26. Chan LK, Chiu YT, Sze KM, Ng IO. Tensin4 is up-regulated by EGF-induced ERK1/2 activity and promotes cell proliferation and migration in hepatocellular carcinoma. Oncotarget. 2015; 6(25): 20964-20976.

27. Wang C, Cigliano A, Jiang L, et al. 4EBP1/eIF4E and p70S6K/RPS6 axes play critical and distinct roles in hepatocarcinogenesis driven by AKT and N-Ras proto-oncogenes in mice. Hepatology. 2015;61(1):200-213.

28. Yang W, Wang C, Lin Y, et al. $\mathrm{OV}^{+}$tumor-initiating cells contribute to tumor progression and invasion in human hepatocellular carcinoma. J Hepatol. 2012;57(3):613-620.

29. Lee $\mathrm{YH}$, Yun $\mathrm{Y}$. HBx protein of hepatitis B virus activates Jak1-STAT signaling. J Biol Chem. 1998; 273(39):25510-25515.

30. Lee YI, Kang-Park S, Do SI, Lee YI. The hepatitis B virus-X protein activates a phosphatidylinositol 3-kinase-dependent survival signaling cascade. J Biol Chem. 2001;276(20):16969-16977.

31. Wang FZ, Fei HR, Lian LH, Wang JM, Qiu YY. Hepatitis B x-interacting protein induces HepG2 cell proliferation through activation of the phosphatidylinositol 3-kinase/Akt pathway. Exp Biol Med (Maywood). 2011;236(1):62-69.

32. Li X, Yang Z, Song $\mathrm{W}$, et al. Overexpression of Bmi-1 contributes to the invasion and metastasis of hepatocellular carcinoma by increasing the expression of matrix metalloproteinase (MMP)-2, MMP-9 and vascular endothelial growth factor via the PTEN/PI3K/Akt pathway. Int J Oncol. 2013;43(3):793-802.

33. Chan C, Wang Y, Chow PK, Chung AY, Ooi LL, Lee CG. Altered binding site selection of $\mathrm{p} 53$ transcription cassettes by hepatitis B virus $\mathrm{X}$ protein. Mol Cell Biol. 2013;33(3):485-497.

34. Hsieh JL, Wu CL, Lee CH, Shiau AL. Hepatitis B virus $X$ protein sensitizes hepatocellular carcinoma cells to cytolysis induced by E1B-deleted adenovirus through the disruption of p53 function. Clin Cancer Res. 2003;9(1):338-345.

35. Chung TW, Lee YC, Ko JH, Kim CH. Hepatitis B Virus X protein modulates the expression of PTEN by inhibiting the function of p53, a transcriptional activator in liver cells. Cancer Res. 2003;63(13):3453-3458.

36. Arima T, Nakao K, Nakata $K$, et al. Transactivation of human alpha-fetoprotein gene by X-gene product of hepatitis B virus in human hepatoma cells. Int J Mol Med. 2002:9(4):397-400.

37. Ogden SK, Lee KC, Barton MC. Hepatitis B viral transactivator HBx alleviates p53-mediated repression of alpha-fetoprotein gene expression. J Biol Chem. 2000; 275(36):27806-27814.

38. $\mathrm{Li} \mathrm{CH}, \mathrm{Xu} \mathrm{F}, \mathrm{Chow} \mathrm{S}$, et al. Hepatitis $\mathrm{B}$ virus $\mathrm{X}$ protein promotes hepatocellular carcinoma transformation through interleukin-6 activation of microRNA-21 expression. Eur J Cancer. 2014;50(15):2560-2569.

39. Xu X, Fan Z, Kang L, et al. Hepatitis B virus X protein represses miRNA-148a to enhance tumorigenesis. J Clin Invest. 2013;123(2):630-645.

40. Sun $Q$, Wang $R$, Luo J, et al. Notch1 promotes hepatitis B virus $X$ protein-induced hepatocarcinogenesis via $W n t / \beta$-catenin pathway. Int J Oncol. 2014;45(4): 1638 -1648.

41. Wang F, Zhou H, Xia X, Sun Q, Wang Y, Cheng B. Activated Notch signaling is required for hepatitis $B$ virus $X$ protein to promote proliferation and survival of human hepatic cells. Cancer Lett. 2010; 298(1):64-73.

42. Uchida T, Takahashi K, Tatsuno K, Dhingra U, Eliason JF. Inhibition of hepatitis-B-virus core promoter by p53: implications for carcinogenesis in hepatocytes. Int J Cancer. 1996; 67(6):892-897.

43. Zhang $\mathrm{C}, \mathrm{He} \mathrm{H}$, Zhang $\mathrm{H}$, et al. The blockage of Ras/ERK pathway augments the sensitivity of SphK1 inhibitor SKI II in human hepatoma HepG2 cells. Biochem Biophys Res Commun. 2013; 434(1):35-41.

44. Xie S, Zhu M, Lv G, et al. Overexpression of Ras homologous C (RhoC) induces malignant transformation of hepatocytes in vitro and in nude mouse xenografts. PLoS One. 2013; 8(1):e54493

45. Liu H, Pan Z, Li A, et al. Roles of chemokine receptor 4 (CXCR4) and chemokine ligand 12 (CXCL12) in metastasis of hepatocellular carcinoma cells. Cell Mol Immunol. 2008; 5(5):373-378.

46. Zhang $\mathrm{C}$, Chen $\mathrm{X}$, Liu $\mathrm{H}$, et al. Alpha fetoprotein mediates HBx induced carcinogenesis in the hepatocyte cytoplasm. Int J Cancer 2015; [Epub ahead of print]

47. Wang S, Jiang $\mathrm{W}$, Chen $\mathrm{X}$, et al. Alpha-fetoprotein acts as a novel signal molecule and mediates transcription of Fn14 in human hepatocellular carcinoma. J Hepatol. 2012; 57(2):322-329.

48. Zheng L, Gong W, Liang P, et al. Effects of AFP-activated PI3K/Akt signaling pathway on cell proliferation of liver cancer. Tumour Biol. 2014; 35(5):4095-4099.

49. Zhang L, He T, Cui H, Wang Y, Huang C, Han F. Effects of AFP gene silencing on apoptosis and proliferation of a hepatocellular carcinoma cell line. Discov Med. 2012;14(75):115-124.

50. Wang XW, Xu B. Stimulation of tumor-cell growth by alpha-fetoprotein. Int J Cancer. 1998; 75(4):596-599.

51. Dai Z, Zhou SL, Zhou ZJ, et al. Capn4 contributes to tumour growth and metastasis of hepatocellular carcinoma by activation of the FAK-Src signalling pathways. J Pathol. 2014; 234(3):316-328.

52. Wang C, Cigliano A, Jiang L, et al. 4EBP1/eIF4E and p70S6K/RPS6 axes play critical and distinct roles in hepatocarcinogenesis driven by AKT and N-Ras proto-oncogenes in mice. Hepatology. 2015; 61(1):200-213.
53. Xiang ZL, Zeng ZC, Tang ZY, et al. Chemokine receptor CXCR4 expression in hepatocellular carcinoma patients increases the risk of bone metastases and poor survival. BMC Cancer. 2009; 9:176 